\title{
DYNAMICAL CONSTRAINTS ON ALTERNATIVES TO MASSIVE BLACK HOLES IN GALACTIC NUCLEI
}

\author{
EYAL MAOZ \\ Astronomy Department, University of California, \\ Berkeley, CA 94720, USA
}

There is now compelling evidence for massive dark objects at the centers of severals galaxies. The possibility that these are black holes (BHs) fits well into the picture where quasars and AGNs are powered by accretion onto a massive $\mathrm{BH}$, so that dead quasar engines should be hiding in many nearby galaxies.

However, as emphasized by Richstone and Kormendy (1995), the massive $\mathrm{BH}$ picture has become a paradigm, which is a dangerous situation since it is easy to believe that we have proved what we expect to find. High $M / L$ ratios in galactic centers do not uniquely imply massive BHs. To argue convincingly that these objects are $\mathrm{BHs}$ we must rule out alternatives to a $\mathrm{BH}$, and the alternative to a point mass is a cluster of some sort of nonluminous stars, such as a cluster of stellar remnants or brown dwarfs. One way to rule out a dark cluster is to show that its lifetime must be very short. A cluster with a lifetime much shorter than $10 \mathrm{Gyr}$ is unacceptable because observing it at the present epoch would be highly improbable.

There are two main process which limit the lifetime of any stellar dynamical system: evaporation, which is the inevitable process of a continuous leakage of stars from the cluster due to weak gravitational scattering, and the destruction of the cluster due to physical collisions between the stars.

A dark cluster may consist of (a) BHs and neutron stars, where the collision timescale is essentially infinite; (b) objects of mass smaller then $\approx 3 \times 10^{-3} M_{\odot}$, where the gravitational forces are small compared with the electrostatic forces (as in planets, for example); and (c) objects which are held by electron-degeneracy pressure, such as brow dwarfs which are hydrogen-rich and thus have masses up to the H-burning mass limit, and white dwarfs.

Since our goal is to rule out a dark cluster by showing that its lifetime must be very short, we must be most generous and assume that the cluster 
may consist of that kind of objects which maximizes its lifetime. Therefore, for every combination of a cluster's half-mass, $M_{h}$, and half-mass density, $\rho_{h}$ (mean density within half-mass radius), we examined all possible stellar types and masses, and found the maximum possible cluster lifetime.

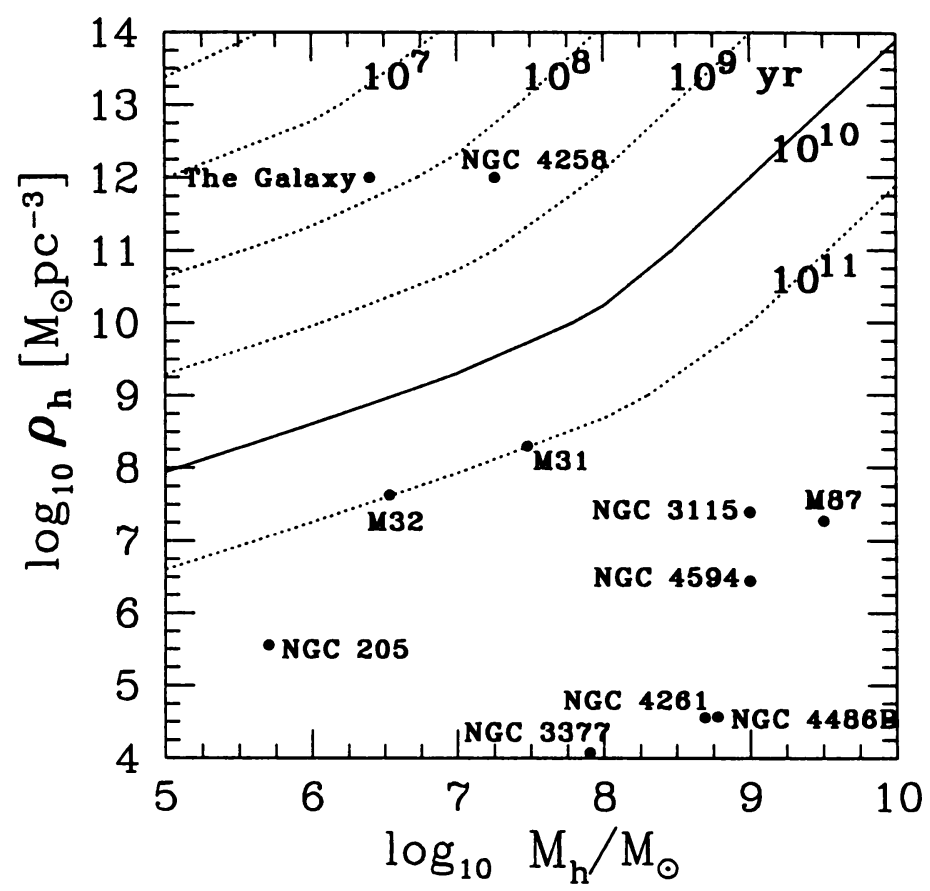

Figure 1. The maximum possible lifetime of a central cluster against the processes of evaporation and physical collisions.

For example, the maximum lifetime of a cluster with half-mass of $10^{7} M_{\odot}$ and half-mass density of $10^{9} M_{\odot} \mathrm{pc}^{-3}$ is $\approx 10^{10}$ years. We note that for different combinations of cluster mass and density, the cluster lifetime peaks at a different stellar type and stellar mass.

We see that only in the cases of NGC 4258 and our Galaxy, the lifetime of an hypothetical central cluster must be much shorter than the galaxy age, thus strongly arguing for a point mass. In all other galaxies, the case of a massive $\mathrm{BH}$, although compelling, is not yet watertight. For the detailed investigation, see Maoz (1997).

\section{References}

Kormendy J. \& Richstone, D. (1995), Inward Bound - The Search For Supermassive Black Holes in Galactic Nuclei, $A R A \& A$, vol. 33, pp. 581-624

Maoz E. (1997) Dynamical Constraints On Alternatives To Massive Black Holes In Galactic Nuclei, submitted to $A p J$ 